\title{
El nuevo enfoque de la política audiovisual de la Unión Europea (1994-1998): la primacía de la dimensión económico-industrial
}

\author{
Carmina Crusafon Baqués \\ Investigadora del Laboratorio de Análisis y Prosprectiva en Comunicación
}

(LAPREC) de Barcelona

La política audiovisual de la Unión Europea ha modificado entre 1994 y 1998 su enfoque principal en respuesta a la nueva situación coyuntural que vive el sector a nivel mundial. Durante este periodo, sus actuaciones han mostrado una primacía económico-industrial (programa MEDIA y el Fondo europeo de garantía) dejando en segundo plano la dimensión cultural ; una mayor coordinación con los proyectos de la sociedad de la información (Libro verde sobre la convergencia) y el mantenimiento un cierto carácter proteccionista (directiva Televisión sin Fronteras y el énfasis en el déficit comercial con los Estados Unidos).

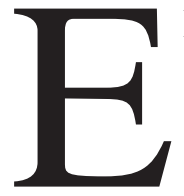
1 escenario de la Unión Europea ha sufrido importantes transformaciones en los últimos cuatro años (1994-1998) en el ámbito político (ampliación y petición de ingreso de nuevos estados, el Tratado de Amsterdam); económico (proceso hacia la unión monetaria) y tecnológico (la llegada de las tecnologías digitales), modificando las actuaciones de los organismos comunitarios. El audiovisual no ha sido ajeno a esta circunstancia, al contrario es un claro ejemplo de los cambios acontecidos. Un análisis de la evolución de la política audiovisual de la Unión Europea durante este periodo pone de manifiesto la adaptación que le ha conducido a primar la dimensión económico-industrial sobre la cultural, así como a plantear los retos a los que se deberá enfrentar en el próximo siglo.

Aunque no tiene tanto peso económico como en los Estados Unidos, la industria audiovisual europea emplea aproximadamente 1'8 millones de personas (COMISIÓN EUROPEA, 1994b:127) y dispone del mercado audiovisual más importante del mundo con 370 millones de consumidores. Por otro lado, las cien primeras grandes empresas audiovisuales en Europa tienen un volumen anual de 7.000 millones de dólares con un crecimiento 
anual del 15\% desde 1995 (OREJA, 1998). A su vez, este sector en Europa se caracteriza por: una fragmentación en mercados nacionales; por un nivel bajo de circulación y distribución transfronteriza de programas; por un déficit crónico y por la incapacidad para atraer recursos financieros para su recuperación.

El carácter dual, económico y cultural, ha caracterizado desde su creación a la política audiovisual europea, con primacía de uno u otro según el periodo analizado. Como afirma Schlesinger (1997b:375), "para los EEUU, el comercio audiovisual es solo un negocio, mientras que para los europeos es tanto un negocio como (cuando conviene) un asunto cultural". Entre 1994 y 1998 la política audiovisual europea ha modificado su discurso en respuesta a la nueva situación coyuntural que vive el sector a nivel mundial. Durante este periodo, su enfoque se ha concretado en: una primacía económicoindustrial (programa MEDIA y el Fondo europeo de garantía), una mayor coordinación con los proyectos de la sociedad de la información (Libro verde sobre la convergencia) y el mantenimiento un cierto carácter proteccionista (directiva Televisión sin Fronteras y el énfasis en el déficit comercial con los Estados Unidos).

Los primeros pasos del nuevo planteamiento audiovisual de la Unión Europea se dieron al tiempo que se concluían las negociaciones de la Ronda Uruguay del GATT a finales de 1993. Sus ideas de base dejaron de lado el aspecto cultural para primar la vertiente industrial, competitiva y de convergencia con las telecomunicaciones. El informe sobre la sociedad de la Información y el libro verde sobre la industria de programas fueron los primeros cimientos. En segundo lugar, la revisión de la directiva « Tlevisión sin Fronteras » y la aprobación de la segunda edición del programa MEDIA, ambos ejes fundamentales de la actuación comunitaria, fueron un paso más hacia una lógica de primacía económico-industrial. Posteriormente, se han elaborado nuevos informes (convergencia de las telecomunicaciones, informática y los medios de comunicación; prospectiva de los mercados audiovisuales en Europa, etc.) en esta misma línea. Todos estos documentos y actuaciones han ido dibujando la imagen de conjunto del audiovisual europeo, poniendo sobre la mesa un nuevo modelo de política audiovisual a nivel de la Unión.

La nueva filosofía de la política audiovisual europea, presente desde la llegada de la Comisión presidida por Jacques Santer, es a grandes rasgos la siguiente: conseguir una industria fuerte, una vez así será posible dar sentido 
a su dimensión cultural y al tiempo crear nuevos puestos de trabajo. Para lograrlo, la política tiene tres pilares: el programa MEDIA, el programa de apoyo a las nuevas tecnologías y la directiva TSF.

\section{Los primeros pasos del nuevo enfoque}

Los objetivos de la política audiovisual de la Unión Europea se centran a partir de 1994 principalmente en la esfera económica con la creación de un mercado interno real, fomentando la competitividad y la presencia europea a nivel global; en la capacidad de dar respuestas al desafío digital y en el fomento de la creación de puestos de trabajo en este sector. Según Collins y Murroni (1996: 122), "el énfasis se sitúa ahora correctamente en la creación de una industria capaz de sostenerse a si misma. Las políticas de apoyo a las producciones culturales son diferentes: no necesitan estar públicamente preocupadas por los temas de eficiencia y deberían permanecer diferenciadas".

La primera nota del carácter económico del audiovisual se presenta en el Libro blanco de Delors (COMISIÓN, 1994b), en el que se detalla las posibilidades de crecimiento y creación de ocupación dentro del sector audiovisual. El Libro Blanco dedica uno de sus capítulos al audiovisual debido a su peso económico y a la importante transformación tecnológica que está experimentando en los últimos años. Califica las perspectivas de crecimiento del sector de "muy espectaculares", a la vez que enfatiza que el audiovisual “posee un notable potencial de creación de ocupación" (COMISIÓN EUROPEA 1994b:127). Afirma que "el sector audiovisual ya no es marginal, ni en términos económicos ni de ocupación. Al contrario, será uno de los principales sectores de servicios en el siglo XXI y se le debe conceder la atención correspondiente" (COMISIÓN EUROPEA 1994b:128).

En la misma época se elabora el informe Bangemann (COMISIÓN EUROPEA, 1994a), dedicado al estudio de la sociedad de la información. Este documento dedica uno de sus apartados a los mercados audiovisuales, señalando algunos de los aspectos que dificultan el desarrollo de esta industria: la falta de una estructura industrial estable, la regulación comunitaria y la concentración de la propiedad. En otras palabras, "nuestro mayor problema estructural es la debilidad financiera y organizativa de la industria europea de programas. A pesar de la enorme riqueza del patrimonio europeo y el potencial de sus creadores, la mayoría de programas y la mayoría de los stocks de derechos adquiridos no están en manos europeas. Un mercado 
nacional fuerte de crecimiento rápido puede suministrar a la industria europea la oportunidad de desarrollar una base doméstica y explotar las posibilidades aumentadas para la exportación. La industria audiovisual de Europa también tiene que cargar con regulaciones. Algunas de ellas quedarán pronto obsoletas por el desarrollo de las nuevas tecnologías, impidiendo el desarrollo de un mercado europeo dinámico" (COMISIÓN EUROPEA, 1994a: 1011). Advierte de la superposición de las regulaciones de ámbito nacional y europeo que se convierten en "un mosaico de inconsistencia que tiende a distorsionar y fragmentar el mercado. Impiden a las compañías aprovecharse de las oportunidades ofrecidas por el mercado interno, especialmente en el multimedia, y pueden ponerlos en peligro frente a los competidores no europeos". Señala como cruciales las normas a nivel europeo, "dada la universalidad de la sociedad de la información y su naturaleza inherentemente transfronteriza. La Unión tendrá que dirigir el camino en la intercepción de la disparidad regulatoria más profunda. Al hacerlo, reforzará la seguridad legal que es vital para la competitividad de la industria europea de los medios" (COMISIÓN EUROPEA, 1994a: 19-20).

Siguiendo en la misma línea de actuación con finalidad a reforzar la estructura industrial del audiovisual europeo, la Comisión Europea encargó a principios de 1994 a un grupo de expertos la elaboración de un informe sobre la política audiovisual (VASCONCELOS, 1994). Se trata del primer paso para la preparación de un Libro Verde sobre la industria de programas audiovisuales. Este documento presenta un diagnóstico de la situación y una serie de recomendaciones para el sector audiovisual europeo. Su situación, pocos meses después de la conclusión de la Ronda Uruguay del GATT, era considerada "grave", a causa de un déficit "colosal y una falta de competitividad".

A partir de un análisis económico y teniendo en cuenta la importancia sociocultural, describe la situación de la industria europea caracterizada por la concepción de las obras audiovisuales destinadas a las audiencia locales ; por la ausencia de redes de distribución transnacionales; y por la existencia de un mercado único que sólo funciona para las industrias no europeas.

El carácter industrial y la concreción son las características de las recomendaciones que da el informe. Sobre la primera, el futuro de la industria se debe estructurar a partir de una estrategia global, que pase de "la lógica de producto a una lógica de mercado" y concebida "según la dimensión y la dinámica futura del mercado europeo, establecidas desde un principio bajo 
la estrategia transnacional y multimedia" (VASCONCELOS, 1994 :35). Esta estrategia se ha de diseñar partiendo de dos directrices. La primera defiende que "las medidas de protección no son sólo necesarias, sino legítimas". La segunda considera primordial "pasar de la cultural a la ofensiva industrial si queremos que nuestra cultura sobreviva" (VASCONCELOS, 1994 :34). Además, la finalidad última tiene que ser una industria fuerte que sea capaz de conseguir mercados para los productos europeos y a la vez crear productos para el mercado. Se recomiendan tres objetivos para la política audiovisual europea: centrarse en el producto o software, en los medios de distribución y en el talento creativo.

Unos meses más tarde, la Comisión elabora el Libro Verde "Opciones estratégicas para el reforzamiento de la industria de programas dentro del contexto de la política audiovisual de la Unión Europea" (COMISIÓN EUROPEA, 1994c). Este Libro Verde pone de manifiesto la importancia estratégica de los contenidos y "propone un replanteamiento de la política audiovisual llevada a término por la Comisión durante la última década" (SÁNCHEZ TABERNERO/ZUNZUNEGUI, 1994: 72).

El Libro Verde, elaborado con la participación de los diferentes sectores, es un documento de discusión, pero a la vez plantea un reto: "la formulación de un sistema de protección que no se convierta en un conjunto de medidas obsoletas al poco tiempo" (SÁNCHEZ TABERNERO/ZUNZUNEGUI, 1994: 73). Su principal objetivo es crear una industria autosostenida y competitiva dentro del mercado internacional, actuando dentro del nuevo marco de la revolución digital interactiva. A la vez reclama una "acción urgente y vigorosa, que pueda permitir a esta industria aprovecharse, en un futuro, del libre juego del mercado" (COMISIÓN EUROPEA, 1994c: 32).

La nueva política europea que propone el Libro Verde ha de basarse en un esfuerzo colectivo con la participación de todos los actores, aprovechando el desarrollo de un espacio europeo de información. Tiene que desarrollar una explotación maximal de los productos y de esta manera, asegurar en un plazo la rentabilidad del sector. El primer esfuerzo a realizar, según el Libro verde, "antes que nada, un cambio de actitud". Es decir, se trata de "reconciliar la cultura y la economía, la obra y el público, las estructuras artesanales y el imperativo industrial" y "de probar la confianza de Europa en sus talentos, de dar con lucidez los medios para la expansión en Europa y alrededor del mundo" (COMISIÓN EUROPEA, 1994c:46).

El Libro Verde sobre la industria de programas es un documento que 
muestra la voluntad de cambio por parte de las autoridades comunitarias con respecto al discurso culturalista mantenido durante las negociaciones de la Ronda Uruguay del GATT. A la vez presenta una propuesta de política audiovisual desde una perspectiva más industrial y económica. Asimismo, deja de lado algunos aspectos, que ha señalado con acierto del dictamen del Consejo Económico y Social (CES 1000/94) (COMITÉ ECONÓMICO Y SOCIAL, 1994). Primeramente, el Libro Verde no desarrolla el riesgo de ver la diversidad de programas y la libertad de expresión amenazadas por los operadores más potentes del mercado internacional. Segundo, deja en silencio el tema de la influencia y los intereses divergentes de los grupos mediáticos que operan a escala mundial. Tercero, las reflexiones sobre la preservación de la especificidad cultural no juegan ningún papel dentro de las "opciones estratégicas".

Por otro lado, como afirma el CES, el Libro Verde obvia las estrategias de limitación de algunas partes del mercado, ya que de esta manera se evitaría una competencia desenfrenada dentro del audiovisual que "acabaría sin ninguna duda con la dominación del mercado europeo por algunos grupos mediáticos, lo más a menudo extranjeros, que constituirían una amenaza para la identidad cultural europea" (COMITÉ ECONÓMICO Y SOCIAL, 1994 :4).Tampoco propone ningún tipo de medidas reglamentarias contra la aceleración del proceso de concentración. En definitiva, el Libro Verde ofrece un nuevo planteamiento para la política audiovisual que deja de lado algunos aspectos como un mínimo control de una competencia ilimitada, el peligro de concentración de la propiedad de la industria, que son elementos básicos para mantener un cierta pluralidad dentro del espacio audiovisual europeo.

Otro de los acontecimientos de esta primera etapa fue la celebración en Bruselas de la Conferencia Europea del Audiovisual (COMISIÓN EUROPEA, 1995a) en el mes de julio de 1994. Este evento se caracterizó, por un lado, por el protagonismo de los profesionales del audiovisual y de otro, por la presentación oficial del nuevo planteamiento de la política audiovisual por parte de las autoridades comunitarias. En la intervención del entonces presidente de la Comisión, Jacques Delors, aunque su parlamento mantuvo dos ideas clásicas del discurso comunitario: la primera, "el bien cultural no es una mercancía como las demás" y la segunda, "preservar la diversidad de nuestras culturas y de nuestras formas de vida", introdujo la necesidad de pensar "en un polo cultural y en un polo industrial" y de tener en cuenta la 
competitividad, el mercado y el progreso técnico (COMISIÓN EUROPEA, 1995a: 10). En la sesión de clausura, el discurso del comisario Joao de Deus Pinheiro fue otra muestra del nuevo planteamiento de la Comisión. "Las acciones futuras para poner en marcha un gran mercado europeo del audiovisual tendrían que tener en cuenta todas las dimensiones - cultural, linguística, económica y tecnológica y reglamentaria- presumiendo que son de naturaleza interactiva y que tienen igual importancia" (COMISIÓN EUROPEA, 1995a: 112). Se presenta una primacía de los aspectos industriales, pero al mismo tiempo mantiene la defensa de la cultura como durante las negociaciones del GATT. Pinheiro afirmó que "la diversidad cultural tiene que ser considerada como un triunfo principal en la formulación de una política mundial del audiovisual" (COMISIÓN EUROPEA, 1995a: 113). En otras palabras, la estrategia a seguir tiene que incorporar la dimensión mundial.

\section{La nueva orientación de los pilares de la política audiovisual común}

La falta de un acuerdo concreto sobre el audiovisual en la Ronda Uruguay del GATT ha permitido a la Unión un periodo de transición para poder reorientar la política comunitaria antes de la llegada de la liberalización completa del sector a nivel mundial. Asimismo se ha producido un cambio en la presidencia de la Comisión con el nombramiento del luxemburgués Jacques Santer y un cambio de carteras entre los comisarios, entre ellas, la del audiovisual pasó a dirigirla Marcelino Oreja . Esta reorientación ha afectado a dos de los pilares de la política audiovisual europea, que han sido revisados: la Directiva «Televisión sin Fronteras» (TSF) y el Programa MEDIA.

La revisión de Directiva «Televisión sin Fronteras» (directiva 97/36/EC) ha supuesto un paso más del nuevo enfoque de la política comunitaria. Es un ejemplo claro de la dificultad de poner de acuerdo las diferentes concepciones sobre el audiovisual que existen entre los distintos estados miembros. Hay que recordar que el proceso de revisión se inició en 1995 con la presidencia francesa de la Unión Europea y no concluyó hasta el 30 de junio de 1997.

Aunque sus rasgos centrales se han mantenido, la revisión ha incorporado algunas provisiones nuevas, entre las que se encuentran: en cuanto a los principios de jurisdicción, se ha clarificado bajo que jurisdicción se debe considerar las actividades de los operadores de televisión, ahora determinadas por donde está localizada su administración central y por donde se to- 
man las decisiones de gestión relativas a la programación. Asimismo se ha introducido una definición de lo que constituye un radiodifusor.

Por otro lado, se han incluido los acontecimientos de gran importancia para el público (particularmente los deportivos): los Estados miembros pueden elaborar una lista de eventos que deben ser retransmitidos en abierto incluso si los derechos de exclusividad han sido comprados por cadenas de televisión de pago. Sobre la base del principio de reconocimiento mutuo, deben asegurar que las diversas cadenas respeten cada una de estas listas. Además se ha ampliado la definición de obra europea para incluir las coproducciones con terceros países. Sobre las producciones independientes, los Estados miembros deben introducir una definición de "productor independiente" para facilitar la aplicación de la norma que requiere el 10\% del tiempo de transmisión o del presupuesto de programación que ha de ser reservado para las producciones independientes. De otra parte, se han abolido los periodos por los cuales los trabajos cinematográficos no podían ser transmitidos en televisión hasta después de haber sido estrenados en salas. Únicamente se requiere a los Estados miembros que aseguren que los periodos acordados entre radiodifusores y los propietarios de los derechos se cumpla.

Las disposiciones sobre la publicidad en televisión continúan siendo las mismas, aunque el límite del $20 \%$ de cualquier periodo de una hora ha sido alterado al $20 \%$ de una hora de reloj dada. La autopromoción se ha asimilado a publicidad y está sujeta a la mayoría de las mismas disposiciones. Además, la telecompra se ha incluido y está sometida a las mismas normas que la publicidad.

El nuevo programa MEDIA (COMISIÓN, 1995b) fue aprobado en 1995 para el periodo 1996-2000. Se estructura en dos líneas de actuación. La primera acción consiste en dar apoyo a la formación, inicial y continua, de los gestores del audiovisual y de los profesionales en gestión económica y comercial, en el uso de nuevas tecnologías y en la escritura de guiones. La segunda tiene como objetivo el desarrollo de producciones europeas y la distribución transnacional de obras audiovisuales. La finalidad del MEDIA II es conseguir resultados más efectivos en la estructuración de la industria audiovisual europea, evitando la dispersión de los efectos que ha causado las 19 líneas de actuación del MEDIA I.

El programa MEDIA II responde a muchas de las peticiones que se habían hecho desde el sector profesional, tanto por parte de los autores del informe sobre el audiovisual, como a las conclusiones de la Conferencia 
Europea del Audiovisual de 1994. Al marcarse solamente tres grandes áreas de actuación, tiene más posibilidades de establecer unos fundamentos sólidos para el desarrollo de una industria audiovisual verdaderamente fuerte y competitiva.

\section{Otras actuaciones a destacar}

Con la voluntad de fomentar una mayor cooperación con el sector de las telecomunicaciones, la DG X y la DG XIII han elaborado el "Libro verde sobre la convergencia de los sectores de las telecomunicaciones, los medios de comunicación y las tecnologías de la información" (COMISIÓN EUROPEA, 1997b). Se trata una muestra del comienzo de la coordinación entre sectores que cada vez están más interrelacioneados pero que hasta entonces estaban poco coordinados en el ámbito comunitario. Este documento "pretende primero y ante todo abrir un amplio debate sobre la convergencia y en particular, sobre la futura evolución del fenómeno y sobre el marco regulador más apropiado para el escenario digital" (PAPPAS, 1998:1). Se enmarca en el contexto de la liberalización de las telecomunicaciones y en el proceso de convergencia tecnológica (aplicación de las tecnologías digitales) e industrial (tendencia hacia las fusiones y alianzas empresariales). Este Libro Verde analiza las barreras existentes y potenciales, que dificultan el proceso de convergencia. De otra parte, señala los retos a los que se enfrenta la reglamentación, entre ellos, la mundialización, una abundancia de ofertas para una reglamentación fundada sobre la penuria, las distinciones entre actividades públicas y privadas y las estructuras reglamentarias. Este documento se encuentra en una fase de debate y estudio por parte de los sectores implicados, antes de que la Comisión emprenda algunas acciones concretas.

En la misma línea de primacía de la dimensión económico-industrial, la Comisión Europea encargó un informe económico sobre el futuro de los mercados audiovisuales (COMISIÓN EUROPEA, 1997b). El propósito del estudio ha sido analizar el efecto económico de las nuevas tecnologías de comunicación en los mercados audiovisuales europeos. En particular, el estudio identifica como las fuentes actuales de ingresos para la industria audiovisual europea pueden crecer en el futuro y como pueden tener un impacto en los productores de contenido audiovisual. El estudio abarca los distintos mercados audiovisuales en siete países de la Unión (Dinamarca, Francia, Alemania, Irlanda, Italia, España y Gran Bretaña) y presenta un análisis integrado de los posibles cambios en todos los segmentos del mer- 
cado audiovisual; al tiempo evalúa las prospectivas de futuro crecimiento a la luz de las diversas demandas del consumidor y del anunciante. En líneas generales, el estudio prevé un crecimiento considerable de los distintos ingresos de los mercados audiovisuales, de los que se espera que aumenten de los 31.800 millones de ECU en 1995 hasta los 53.900 millones de ECU en el 2005 (según los precios de 1995) (COMISIÓN, 1997b:4).

Otro aspecto de la política europea a señalar es la adopción de una propuesta de recomendación sobre la protección de los menores y de la dignidad humana (COMISIÓN EUROPEA, 1997c). Esta propuesta en si misma pretende promover unas directrices comunes para la implementación, a nivel nacional, de un marco de auto-regulación para proteger menores y la dignidad humana en los servicios audiovisuales y de información, cualquiera que sea el medio de distribución.

Por otro lado, el comisario Oreja ha creado un Grupo de alto nivel para la política audiovisual con la finalidad que le asesore ante el nuevo escenario audiovisual. Además, se celebrará la Conferencia Europea del Audiovisual bajo la presidencia británica, durante el mes de abril de 1998, con el objeto de evaluar los desafío que plantean las tecnologías digitales al sector audiovisual europeo. La conferencia ha centrado sus sesiones de trabajo en cuatro grandes áreas temáticas: el empleo, el apoyo europeo al sector audiovisual, el marco adecuado para una economía de los medios de comunicación creativa en una sociedad democrática y la propiedad intelectual.

A pesar de la primacía de la dimensión económico-industrial durante el periodo analizado, existe una cierta continuidad en algunas de las ideas que constituyen las ideas de fondo de la política audiovisual europea. De un lado se mantiene el carácter proteccionista de la política de la Comisión Europea; principalmente se observa en la revisión de la directiva "Televisión sin Fronteras", que mantiene el concepto de las cuotas, y en el énfasis que se dedica al déficit comercial entre la UE y los EEUU en los productos audiovisuales. Esta concepción de carácter proteccionista tiene ciertamente una base real porque el déficit de la balanza comercial está creciendo a un ritmo más rápido que anteriormente, pasando de un $11 \%$ de incremento en 1995 a un $18 \%$ en 1996. Este último año ha alcanzado un total de 5.600 millones de dólares y se espera que llegue a un nivel no menor al 9\% en 1997, a juzgar a partir de las indicaciones preliminares (OBSERVATORIO AUDIOVISUAL EUROPEO, 1998). Con estos datos, las instancias comunitarias pueden reforzar la idea de una Europa unida fuerte así como presen- 
tar la europeización "como una clase de antídoto ante una amenaza externa" (SCHLESINGER, 1997b:373). Principalmente, esta concepción es defendida por Francia y los países del área mediterránea, que tienen tradición de apoyo gubernamental a esta industria. Sin embargo, hay que señalar que este enfoque choca con otras iniciativas elaboradas desde otras dependencias de la Comisión, que plantean proyectos como la creación del mercado transatlántico (fruto de la Nueva Agenda Transatlántica) o incluso con el espíritu de cooperación que ofrecen algunos discursos del comisario Oreja (OREJA, 1998).

Por otro lado, se observa un progresivo abandono de los métodos microeconómicos por un enfoque macroeconómico y "desde las negociaciones del GATT, el euro-discurso oficial dominante ha pasado de la defensa de la identidad colectiva a la creencia que el verdadero camino para los valores culturales yace en el consumo individualizado" (SCHLESINGER, 1997a: 5). De esta manera, "la nueva fórmula mágica parece ser: la compresión digital más la euro-heterogeneidad igual a rentabilidad, además de asegurar la competitividad global" (SCHLESINGER, 1997b: 380).

\section{Conclusiones}

Ante la nueva situación mundial del audiovisual, la política europea ha necesitado redefinir sus actuaciones. Su opción ha sido primar la dimensión económico-industrial para conseguir a posteriori los objetivos culturales y de empleo. Este cambio de discurso debe ser considerado positivo si se alcanza el objetivo central de consolidar la base industrial del sector. Por este motivo sus resultados deben primero evaluarse desde la perspectiva económica y desde el punto de vista de la consolidación de un verdadero mercado común para la industria europea. Por otro lado, si la primacía de lo económico significa dejar de lado la dimensión cultural, ¿cómo se conseguirá avanzar en la creación de una verdadera identidad europea?. El audiovisual es un buen medio para configurar una base común a todos los europeos, si se fomenta solo la dimensión económica este aspecto tenderá a perderse y la Unión Europea se quedará en algo simplemente económico sin bases culturales comunes, hecho que se agudizará con la llegada de nuevos Estados miembros en el siglo XXI.

Además, la política audiovisual europea tiene ante si el reto de la globalización de la economía y el dominio de los grandes grupos multimedia, que le obligan a actuar sin dilación, a adaptarse al cambio espacial de la 
industria audiovisual y a un escenario cada vez más liberalizado. Por esta razón, la política audiovisual comunitaria necesita coordinar sus actuaciones en dos niveles: el primero, la incorporación dentro de su plan de acción los distintos ámbitos del espacio audiovisual europeo teniendo en cuenta el principio de subsidiariedad ; y segundo, estructurar una actuación conjunta europea que le permita competir en la esfera global en igualdad de condiciones que la industria estadounidense y japonesa.

Una de las posibles consecuencias de la primacía de este enfoque económico-industrial puede ser una concentración en la propiedad de la industria audiovisual en detrimento del pluralismo y la diversidad, tema esencial para el desarrollo completo de una sociedad democrática. Pero este aspecto no parece estar entre las prioridades de los responsables de la política audiovisual europea, ya que desde la elaboración en 1992 del Libro Verde sobre el pluralismo, no han aparecido documentos sobre esta temática. Sin embargo, hay que señalar que este es uno de los aspectos a los que se debería prestar atención para evitar que más tarde el dominio empresarial esté tan consolidado que haga en valde cualquier actuación comunitaria.

Para concluir, no hay que olvidar que el audiovisual es uno de los sectores con mayores perspectivas de creación de empleo, con unas expectativas de creación del orden de 2 millones de puestos de trabajo para el año 2000 (COMISIÓN, 1994b :127), por lo que la política audiovisual de la Unión no debe desaprovechar esta oportunidad, ni tampoco dejar de reforzar los vínculos entre esta industria y la sociedad de la información. De esta manera conseguirá establecer unos buenos cimientos para mantener a la Unión Europea como uno de los bloques económicos fuertes en la economía del siglo XXI. 


\section{Bibliografía}

- COLLINS, Richard: Broadcasting \& Audio-visual Policy in the European Single Market, John Libbey, Londres, 1994.

- COLLINS, Richard y Cristina MURRONI: New Media. New Policies. Media \& Communications Strategies for the Future, Polity Press, Cambrige, 1996.

- COMISIÓN EUROPEA: Green Paper on the Convergence of the Telecommunications, Media and Information Technology Sectors, and the implications for regulation. Towards an Information Society Appproach, COM(97) 623, 3 de diciembre, Bruselas, 1997a.

- COMISIÓN EUROPEA: Economic Implications of New Communication Technologies on the Audio-Visual Markets, final report, marzo, Bruselas, 1997b.

- COMISIÓN EUROPEA: La industria cinematográfica a examen. Segundo informe 1997, DG X/C, Bruselas, 1997c.

- COMISIÓN EUROPEA: L'industrie europpéenne du cinéma en analyse. Premier rapport d'information 1996, DG X/D, noviembre, Bruselas, 1996 .

- COMISIÓN EUROPEA: Comunicación sobre los servicios de interés general en Europa, COM (96) 443 Final, 11 de septiembre, Bruselas, 1996 b.

- COMISIÓN EUROPEA: "Vivir y trabajar en la sociedad de la información: prioridad para las personas. Libro verde", Boletín de la Unión Europea, Suplemento 3/96, Bruselas,1996c.

- COMISIÓN EUROPEA: Actes de la Conférence europénne de l'audiovisuel. Bruxelles, les 30 junin, ler et 2 juillet 1994, CECA-CECEEA, Bruselas, 1995a.

- COMISIÓN EUROPEA, Propuesta de deicisión del consejo relativa a un programa de formación para los profesionales de la industria europea de programas audiovisuales (Media II-Formación) (1996-2000) y propuesta de decisión del Consejo relativa a un programa de estímulo al desarrollo y a la distribución de obras audivosuales europeas (Media II- Desarrollo y distribución) (1996), COM(94) 523 final -95/0026(SYN), Bruselas, 1995b.

- COMISIÓN EUROPEA: Europe and the global information society. Reccommendations to the European Council, mayo, Bruselas, 1994a.

- COMISIÓN EUROPEA: Crecimiento, creatividad y empleo. Retos y pistas para entrar en el siglo XXI. Libro blanco, CECA-CE-CEEA, Bruselas, 1994b. 
- COMISIÓN EUROPEA: Opciones estratégicas para el reforzamiento de la industria de programas dentro del contexto de la política audiovisual de la Unión Europea. Libro Verde, CECA-CEE-CEEA, Bruselas, 1994c.

- COMITÉ ECONÓMICO Y SOCIAL DE LAS COMUNIDADES EUROPEAS: Avis sur le Livre Vert relatif aux "Options stratégiques pour le renforcemente de l'industrie des programmes dans le contexte de la politique audiovisuelle de l'Union europénne" CES1000/94 D-VN/SL/ct, 14-15 septiembre, Bruselas, 1994.

- GAURON, André: "La place de la culture et des médias. L'identité au péril du marché”, Médiaspouvoirs, núm. 39-40, Paris, 1995, pp.256-263.

- HUMPHREYS, Peter J.: Mass Media and Media policy in Western Europe, Manchester University Press, Manchester, 1996.

- KAITATZI- WHITLOCK, Sophia: "Pluralism and Media Concentration in Europe. Media policy as Industrial Policy", European Journal of Communication, vol. 11, núm. 4, pp.453-483.

- MATTELART, Armand: "Excepción o especificidad cultural. Los desafíos del GATT", Telos, núm.42, 1995, Fundesco, pp.15-27.

- OREJA, Marcelino: "To strengthen the competitiveness of European audiovisual industry and raise the profile of our cultural and linguistic diversity", discurso en el American Film Market, 2 de marzo, Santa Mónica (EEUU), 1998.

- OREJA, Marcelino: “Audiovisual policy: progress and prospects”, memo, Bruselas, 1997.

- PAPPAS, Spyros A: "Presentation of the Green Paper on the Convergence of the Telecommunications, Media and Information Technology Sectors and the Implications for Regulation", discurso, 13 de enero, Bruselas, 1998.

- RELJIC, Dusan: "Muzzling the nationalist monster: can the media make us more European?", The Bulletin, vol. 14, núm.4, European Institute for the Media, Düsseldorf, 1997, pp.9-10.

- SÁNCHEZ TABERNERO, Alfonso y Santos ZUNZUNEGUI: « La política audiovisual europea », SITUACIÓN, Servicio de Estudios del BB》 n³, Bilbao, p.53-77.

- SCHLESINGER, Philip: "Building a collective European identity through the Media”, The Bulletin, , vol. 14, núm. 3, Düsseldorf, European Institute for the Media, 1997a, pp.4-5. 
- SCHLESINGER, Philip: "From cultural defence to political cultural: media politics and collective identity in the European Union", Media, Culture \& Society, vol.19, Sage, Londres, 1997b, ppp.369-391.

- SCHLESINGER, Philip: “¿Debemos preocuparnos por Norteamérica? La ppolítica cultural y audiovisual de la Unión Europea”, Telos, núm.4, mayo marzo, Fundesco, 1995, pp.17-28.

- VAN HEMEL, Annemoon: "The complex debate on audiovisual policy", en VAN HEMEL, Annemoon, Hans MOMMAAS Y Cas SMITHUÏJSEN (eds): Trading Culture: GATT, European Cultural policies and the transatlantic market, Boekman Foundation, Amsterdam, 1996, pp.83-95.

- VASCONCELOS, Antonio-Pedro (dir): Rapport de la cellule de réflexion sur la politique audiovisuelle dans l'Union europénne, CECA-CE-CEEA, Bruselas, 1994. 